\title{
Comparison of PET.CT and MRI imaging Scans Detection Methods for the Diagnosis of Gastric Cancer
}

\author{
Nouradin Hashemi and Javad Haddadnia* \\ Medical Engineering Department of Hakim Sabzevari University, Iran. \\ DOI: http://dx.doi.org/10.13005/bbra/1896
}

(Received: 20 September 2015; accepted: 04 November 2015)

\begin{abstract}
Cancer is a disease that human being has been suffering from it for a long time. Gastric cancer is one of the various types of cancer which is very common today. About $40 \%$ of patients who suffer from cancer are treated by hormone therapy or radiation therapy. In this method cancer cells are destroyed using methods PET.CT or MRI imaging scans. The process is done using two methods of external radiation therapy and internal radiation therapy. In cancer treatment by these methods, the ultimate goal is to deliver maximum dose to cancerous tissue with minimal side effects on healthy tissue around the tumor. Advances in technology have helped that the field of rays be limited to the treatment area as far as possible and thereby the healthy tissues are largely protected. The use of MRI in medicine, particularly in the field of cancer diagnosis and treatment has attracted many researchers over the past two decades and is still being used to reduce the damages and to prevent the spread of disease symptoms (such as pain). These devices are essentially used to identify and locate the related tumor and the tissue receives different types of rays, the most common of which include the computed tomography (CT), magnetic resonance imaging (MRI) and recently introduced positron emission tomography (PET). After identifying the desired tissue location, the simulation should be started which is planned based on the anatomy of the patient. This paper tries to compare PET.CT and MRI imaging scans detection methods for the diagnosis of gastric cancer.
\end{abstract}

Keywords: Gastric cancer, PET.CT method, tomography, MRI

The increase in mortality rates for gastric cancer represents a growing trend of the disease so that, following lung cancer, gastric cancer is the second most common cancer in men and the second leading cause of cancer death. If radiation therapy is necessary to treat this cancer, using new methods will be effective and play an important role in the treatment of this disease and reduces the exposure of nearby healthy tissue and reduces side effects of radiation treatment.

Today, new methods of the adaptive three-dimensional conformal radiation therapy (3DCRT) are used in the most advanced medical

\footnotetext{
* To whom all correspondence should be addressed.
}

centers in Iran and abroad. With the advances in the adaptive three-dimensional conformal radiation therapy (3DCRT) in recent years, significant changes have occurred in gastric cancer treatment planning. One of the most important parameters in radiation is to determine the volume of the target, the results of which are used to plan the treatment of tumors. Today, different techniques of magnetic resonance imaging (MRI) are being increasingly used for the detection of cancers.

In many radiotherapy centers, the combination of MRI images and CT are used to define and determine the target volume. However, using the technique of magnetic resonance spectroscopy (MRS) is a method of interest for oncologists in the definition of the target volume. In addition to CT and MRI magnetic resonance 
which provide the morphological data of the tissue containing tissue biochemical information. Since the biochemical activity of tumors is different from normal tissue, magnetic resonance spectroscopy (MRS) has a specific potential in the diagnosis of tumors which lack sufficient contrast with morphological images. One of the distinguishing characteristics of gastric carcinoma is the multifocus and its coexistence at different parts of stomach and spreading out to the capsules so that the centers are not detectable by CT and MR imaging.

CT, MRI and magnetic resonance spectroscopy were prepared for a total of 20 patients with gastric cancer referring to the radiotherapy. Magnetic resonance spectrometry (MRS) was prepared using spin-echo 3D CSI protocol with a Siemens 5.1 Tesla MRI system model. The anorectal coil was used to increase the signal to noise ratio. TR and TE values were chosen $120 \mathrm{~m}$ and 1300 seconds, respectively. The number of stimulation (NEX equal to 4 and voxel about 0.45 cubic centimeter, and the desired volume (VOI) was set and placed exactly on the stomach and excitation and signal interference outside the area was prevented using pre-impregnated bands.

In the T2 MRI images, Turbo Spin Echo protocol was used with 4200 and 108 milliseconds TR and TE respectively and ETL $=32$, the thickness of area was selected as $3 \mathrm{~mm}$ with $0.3 \mathrm{~mm}$ distance between the sections, matrix size of $256 \times 205$ and $200 \mathrm{~mm}$ field of view (FOV). The CT protocol for Pelvic RT stored on the system was used in order to simulate and get the images on the system. In this Protocol the voltage was set to $120 \mathrm{kV}$ and effective $\mathrm{mA}$ was set as $250 \mathrm{~mA}$ and a thickness of $3 \mathrm{~mm}$.

For treatment planning, CT and MRI images are entered into the fusion system as DICOM and are fused in Core fusion software. Then based on ICRU definition, due to the multifocus of adenocarcinoma of the stomach, the entire stomach is considered as gastric target tumor volume (GTV). But the main focus of this study was to determine the clinical target volume (CTV). In the conventional treatment planning using CT images the entire stomach with 0.5 inch margin as well as the seminal vesicle is considered as CTV.

In planning treatment with MRI image stomach and seminal vesicle with a margin of 0.5 $\mathrm{cm}$ in volume are considered. In planning with magnetic resonance spectrometry images (MRSI), the images of the stomach are added to the volume clinically identified in CT and thus three types of clinical target volumes contains images of magnetic resonance spectroscopy after conversion to DICOM files were fused on CT images and for treatment plan, were entered into Core plan treatment planning system.

The resulted MRI-CTV, MRI-CTV and planning target volume (PTV) were also drawn by adding $0.5 \mathrm{~cm}$ margin around and $0.5 \mathrm{~cm}$ posterior to CTV and then organs at risk were contoured. From among five treatment fields with the incidence angle of 0, 90, 120, 240 and 270 degrees with an energy dose of 15 Megavoltage in total 7000 cGy were used in 35 sessions.

CT-CT of all the volume in this study are provided in cc and dose value as a percentage of prescribed doses, that is repeated measures of a specified variable values in several sampling. This definition is the generalized mode of paired t-test. However, in the paired t-test a group is compared in two states while in a repeated measures plan a group is compared in two or more conditions.

In most disorders and diseases before anatomical changes of the tissue, some changes occur in the metabolism. Thus, unlike the conventional imaging systems of CT and MRI that measure anatomical changes or SPECT that can measure physiological changes, using PET system make it possible to recognize the disease at an early stage and prevent disease progression and deal with its treatment and as it is known early diagnosis is critical for cancer treatment. It is often observed that after surgery of brain tumors, tumor grows and puts the patient in trouble once again.

It is due to the fact that by using existing imaging systems, one can only detect the tumor mass and the surgeon removes the tumor mass only by using these images while by using PET, not only tumor position is determined but the spot and advance direction are also specified. As it is known, the glucose metabolism rate increases in cancerous tissue. So, the tissues diagnosed with cancer cells though may not be detected with other imaging systems but are detectable by PET and metabolism check. Therefore, the surgeon may also be able remove the tissue on time.

PET system can be used for evaluating 
the selected therapy for patients with cancer. Observing the treatment effects of radiotherapy and chemotherapy as anatomical changes in tumor require a long time to be recognized by conventional imaging methods, while if proper treatment is prescribed the reduction of tumor glucose metabolism can be observed after a short time by PET.

In contrast, if there is no indication that metabolism is reduced, the prescribed treatment is not effective and another way must be selected for treatment. Information accessed when the PET is used are crucial for saving the patient life and PET systems have a significant advantage in the diagnosis of cancer, particularly the secondary cancers (meta stasis) over other imaging systems and the following charts indicate the superiority of PET detection system to CT scans for lung and colon cancer detection and mammography device to detect breast cancer.

PET has a significant advantage in the detection of metastatic foci (secondary cancer) compared to other diagnostic devices and by scanning the entire body by PET and realizing multiple foci of second cancers in the body, the need for surgery to remove the primary tumor mass is changed and the additional costs and unnecessary surgery are prevented.

PET can be used to assess blood flow and metabolism in the heart muscle, so PET is the most accurate system to detect the presence or absence of coronal disease and at the same time by examining the metabolism of the heart muscle, one can find if the tissue is living. If the PET system is used to examine the metabolism of heart muscle in patients whose part of heart muscle is dead and bypass surgery and blood supply to the heart muscle have had no benefit for them before surgery, a major surgery with its risk causing unnecessary patient suffering and costs can be avoided.

So using PET images not only those patients with heart disease whose problem was not solved by surgery can be separated from others but it becomes possible to evaluate the success rate of surgery after the surgery. Due to decreased metabolism in parts of the brain in the early stages of the disease, PET scans can detect the Alzheimer disease several years before doctors can detect it by conventional methods". PET can detect Parkinson's disease better than the other imaging systems. The brain surgeons only by help of the PET images can identify the precise location of seizure foci in the brain, remove them and treat the patient.

Regarding the PET diagnostic capabilities, a PET center should be opened in Iran to provide advanced medical services and help to increase the level of treatment in the country and avoid sending patients abroad. Cost-effectiveness of prescribing PET imaging for a group of patients is so that in many developed countries, insurance organizations cover PET scan costs between US \$ 1800 to 2000 to reduce the cost of treatment. In short, a PET system is effective not only in saving health services but also using this system can save human lives.

\section{RESULTS}

The statistical analysis of the results shown in three methods of treatment

The results of statistical analysis showed significant differences in the PTV $(\mathrm{P}<0 / 0001)$ and CTV $(\mathrm{P}<0 / 0001)$. The results of statistical analysis to compare the volume of GTV $(\mathrm{P}=0 / 699)$, bladder $(\mathrm{P}=0 / 141)$, rectum $(\mathrm{P}=0 / 566)$ and the femur $(\mathrm{P}=0$ / 204) indicated a lack of significant difference in the use of the above three methods.

MRI and magnetic resonance percentage volume changes compared to CT using is obtained by equation 1 . The mean percentage volume changes and CTV and PTV in MRI compared to CT were $83 / 12 \%$ and $97 / 8 \%$ less, respectively. The mean CTV and PTV percentage change in MRS compared to CT were $21 \%$ and $41 / 27 \%$ more, respectively.

The maximum dose received by the organs at risk

The maximum dose received by the organs at risk were extracted from DVHs. The results of the statistical analysis showed no significant differenc e in maximum dose received by the bladder $(\mathrm{P}=0 / 141)$. However, the amount in the rectum $(\mathrm{P}=0 / 02)$ and the femur $(\mathrm{P}=$ $0 / 003$ ) were significantly different. The mean percent change in dose received by the rectum represents 58/0\% increase of this value in MRS compared to CT and 09/1\% reduction in MRI compared to CT. The maximum dose in the femur increased 4/5 percent in MRS compared to CT and 
showed 67/0\% reduction in MRI method compared to $\mathrm{CT}$.

The standard deviation of dose was used in order to evaluate the uniformity of the received dose distribution to the treatment volumes in GTV, CTV and PTV. The results of statistical analysis showed significant differences between the standard deviation of the dose absorbed to the PTV ( $=0 / 007)$, which indicates the uniformity of the dose distribution in the target volume. The mean percent change in dose standard deviation was 95/4\% more in PTV MRI compared to CT and 28/18\% higher in MRS compared to CT. This amount on CTV MRI compared to CT and in MRS compared to CT showed $05 / 1 \%$ and $06 / 15 \%$ increase, respectively.

\section{DISCUSSION}

In general, one of the most important points in adaptive treatment planning is the precise clinical definition of target volume and organs at risk. The use of CT increases the accuracy of the radiation dose and the target volume while at the same time it has some limitations. In planning adaptive treatment for gastric cancer, there are some limitations for the definition of treatment with the use of CT. CT images is limited in comparison with MRI due to the low contrast and resolution and lack of differentiation between soft tissues around the stomach and the stomach, especially in the determination of the head of the gland.

This limitation is due to the relatively similar X-ray attenuation at different soft tissues that leads to a lack of sufficient contrast between the adjacent anatomical structures. In addition, in CT images, there is a partial volume effect that causes lack of delineation of various tissues. MRI images compared to CT have higher contrast in showing the soft tissues and using it in many cases helps to determine more accurate treatment volume and organs at risk. Most applications of MRI for treatment planning in radiotherapy are in form of fusion with CT to overcoming the limitations of MRI images.

Some of these limitations have been reported including the distortion of the images and the absence of physical information, such as the electron density of the tissues. Using MRI may determine the exact number of difficulties, including the head of the stomach in determining target volume for radiation therapy, which is resulting in increasing treatment. According to the discussion above, in the present study, the use of MRI in defining the target volume reduced the target volume due to the high contrast images.

In other studies, using MRI image has reduced the target volume. As Austin Becker et al (2003) in their study showed reduction of the volume to 4/1 CT volume. In another study, Jackson et al (2007) investigated the use of MRI images in the design and evaluation of gastric stomach volume in this study indicated significant differences in the definition of the stomach volume using CT and MRI. The average size of the stomach was about $38 \pm 14 \mathrm{ml}$ by CT and $33 \pm 13 \mathrm{~mL}$ using MRI.

Many studies have attributed the difference in defining the stomach volume to good contrast resolution of MRI images. But an ideal imaging technique for the determination of the target volume must have high sensitivity and specificity to be able to provide sufficient information to draw a precise definition of the tumor, including the tumor spread outside of the anatomical range. The study of Zapatazna et al showed that the sensitivity and specificity of magnetic resonance to assess the extent and volume of gastric adenocarcinoma was reported between 5 / 38-77\% and 5 / 38-78\% so that the combination of MRI with MRS increases the value to about $100 \%$. The magnetic resonance imaging (MRS) is one of the biological methods that are non-invasive and has the potential to change the radiation therapy by precise determination of tumor.

The areas where the spectrum peak of citrate / choline + Keratin is higher than the ratio in healthy areas can be considered as a biological target volume (BTV). It is suggested that maximum dose is applied to this volume. The metabolic ratio can be used to determine the operating position of the tumor in the stomach. Thus the use of magnetic resonance to determine areas of cancer from healthy areas in determining the target volume increases the treatment ratio. Many studies also introduced the use of magnetic resonance as a method to increase the accuracy of measurements of gastric cancer spread outside the capsule.

So that in 2010, Hu Jing Zhang et al assessed the validity of the tumor spread ration 
using magnetic resonance in patients with T1c phase gastric cancer. The results of the histopathological findings were compared with magnetic resonance and the accuracy of the method was estimated over $80 \%$. The studies conducted by Schidler in 1999 and 2010 also introduced MRS as a good method for determining the position of the tumor, the volume of the tumor and the tumor stage. Sarhanj et al also stated that the use of magnetic resonance spectroscopy in determining the spread of cancer outside the capsule reduces the differences between the radiologists.

The results showed an increase in the target volume using magnetic resonance compared to CT that can be because of the ability magnetic resonance spectroscopy to detect and determine the active tumor out of the stomach. As a result, use MRS increased the clinical target volume. Increases and decreases in the maximum dose reached to the organs at risk is also influenced by the size of the field and the volume of treatment. Thus, the MRI reduces the target volume and MRS increases the target volume and also the absorbed dose to organs at risk is decreased and increased, respectively.

In addition, the standard deviation of the dose in the target volume as an indicator of the uniformity of the dose distribution can be considered as a function of the target volume and content uniformity and regularity in determining its borders. According to research findings, it can be concluded that the use of magnetic resonance for treatment planning needed further studies including the resolution protocol used in this method which affect the target volume. The post processing actions are also considered to produce metabolites affected by the selected voxel on the desired area.

\section{REFERENCE}

1. Cotran RS, Kumar V, Collins T. Robbins pathologic basis of disease: Saunders Philadelphia 1999.

2. Debois M, Oyen R, Maes F, Verswijvel G, Gatti $\mathrm{G}$, Bosmans $\mathrm{H}$, et al. The contribution of magnetic resonance imaging to the three dimensional treatment planning of localized prostate cancer. Int J Radiat Oncol Biol Phys. 1999; 45(4):85765.
3. Jackson AS, Reinsberg SA, Sohaib SA, CharlesEdwards, EM, Mangar SA, South CP, et al. Distortioncorrected, T2 weighted MRI: a novel approach to prostate radiotherapy planning. Br J Radiol 2007; 80(959):92633.

4. Khan F. Treatment planning in radiation oncology: Lippincott Williams \& Wilkins 2007.

5. Khoo VS, Padhani AR, Tanner SF, Finnigan DJ, Leach MO, Dearnaley DP. Comparison of MRI with CT for the radiotherapy planning of prostate cancer: a feasibility study. Br J Radiol. 1999; 72(858):5907. Roach M 3rd, Faillace Akazawa

6. N, Bock T, Goldner G, Kovacs G, Fransson A, et al. Interobserver comparison of CT and MRIbased prostate apex definition. Clinical relevance for conformal radiotherapy treatment planning. Strahlenther Onkol. 2002; 178(5): 2638.

7. P, Malfatti C, Holland J, Hricak H. Prostate volumes defined by magnetic resonance imaging and computerized tomographic scans for three dimensional conformal radiotherapy. Int J Radiat Oncol Biol Phys. 1996; 35(5):10118. Wachter S, Wachter Gerstner

8. Parkin DM, Bray F, Ferlay J, Pisani P. Global cancer statistics, 2002. CA Cancer J Clin. 2005; 55(2):74108.

9. Payne GS, Leach MO. Applications of magnetic resonance spectroscopy in radiotherapy treatment planning. Br J Radiol. 2006; 79 Spec No 1:S1626.

10. Steenbakkers RJ, Deurloo KE, Nowak PJ, Lebesque JV, van Herk M, Rasch CR. Reduction of dose delivered to the rectum and bulb of the penis using MRI delineation for radiotherapy of the prostate. Int J Radiat Oncol Biol Phys. 2003; 57(5):126979.

11. Villeirs GM, De Meerleer GO. Magnetic resonance imaging (MRI) anatomy of the prostate and application of MRI in radiotherapy planning. Eur J Radiol. 2007; 63(3):3618.

12. Villeirs GM, L Verstraete K, De Neve WJ, De Meerleer GO. Villeirs G. Magnetic resonance imaging anatomy of the prostate and periprostatic area: a guide for radiotherapists. Radiother Oncol. 2005; 76(1):99106.

13. Yuen JS, Thng CH, Tan PH, Khin LW, Phee SJ, Xiao D, et al. Endorectal magnetic resonance imaging and spectroscopy for the detection of tumor foci in men with prior negative transrectal ultrasound prostate biopsy. J Urol. 2004; 171(4): 14826.

14. Zapotoczna A, Sasso G, Simpson J, Roach M. Current role and future perspectives of magnetic 
resonance spectroscopy in radiation oncology for prostate cancer. Neoplasia. 2007; 9(6): 45563.

15. Zhang J, Hricak H, ShuklaDave, A, Akin O, Ishill NM, Carlino LJ, et al. Clinical stage T1c prostate cancer: evaluation with endorectal MR imaging and MR spectroscopic imaging. Radiology. 2009; 253(2):42534. 